(c) 2020 Universidad Nacional Autónoma de México, Facultad de Estudios Superiores Zaragoza.

Este es un artículo Open Access bajo la licencia CC BY-NC-ND (http://creativecommons.org/licenses/by-nc-nd/4.0/).

TIP Revista Especializada en Ciencias Químico-Biológicas, 23: 1-18, 2020.

https://doi.org/10.22201/fesz.23958723e.2020.0.261

\title{
Utilización de subproductos agroindustriales para la bioadsorción de metales pesados
}

\author{
1a Jonathan M. Sanchez-Silva, **1b Ramsés R. González-Estrada, \\ ${ }^{1 b}$ Francisco J. Blancas-Benitez y *1a Ángel Fonseca-Cantabrana \\ ${ }^{a}$ Departamento de Ingeniería Química y Bioquímica, bivisión de Estudios de Posgrado \\ e Investigación, ${ }^{1}$ Tecnológico Nacional de México/ Instituto Tecnológico Tepic. \\ Av. Tecnológico \# 2595, Col. Lagos del Country, Tepic 63175, Nayarit, México. \\ E-mails:*afonseca@ittepic.edu.mx**ramgonzalez@ittepic.edu.mx
}

\begin{abstract}
RESUMEN
La contaminación por metales pesados es un problema que afecta a los ambientes acuáticos y terrestres, y cuya principal fuente son las actividades antrópicas. Para atender este problema, la comunidad científica ha desarrollado métodos físico-químicos para la remoción de metales pesados en efluentes contaminados: sin embargo, la mayoría no son económicamente favorables, ya que presentan elevados costos de operación y mantenimiento, además de que algunos generan residuos difíciles de manejar. Sin embargo, existe un método de bajo costo, altamente eficiente y sin formación de contaminantes secundarios, denominado bioadsorción. La bioadsorción utiliza subproductos agroindustriales con el objetivo de utilizar la excesiva generación de estos residuos como bioadsorbentes, para la remoción de metales pesados en aguas residuales. La utilización de subproductos agroindustriales como bioadsorbentes ha mostrado ser una alternativa para su aprovechamiento, consecuentemente, México tiene potencial en la producción de bioadsorbentes. El objetivo de esta revisión es proporcionar información sistematizada del método de remoción de metales pesados por bioadsorción a través del uso de subproductos agroindustriales.
\end{abstract}

Palabras clave: bioadsorción, metales pesados, subproductos, contaminación, agroindustria.

Biosorption of heavy metals by agroindustrial byproducts

\begin{abstract}
Heavy metal pollution is a problem that affects aquatic and terrestrial environments, where the main source is anthropic activities. To attend this problem, the scientific community has developed physical-chemical methods for the removal of heavy metals in contaminated effluents: however, they are not economically favorable as they have high operating and maintenance costs, and some generate waste that is difficult to handle. However, there is a low-cost, highly efficient method without the formation of secondary contaminants, called bioadsorption. Bioadsorption uses agro-industrial by-products with the objective to use the excessive generation of these waste as bioadsorbents for the removal of heavy metals in wastewater. The use of agro-industrial by-products as bioadsorbents has proven to be an alternative for their use, consequently, Mexico has potential in the production of bioadsorbents. The objective of this review is to provide systematized information on the method of removing heavy metals by bioadsorption through the use of agro-industrial by-products.
\end{abstract}

Keywords: biosorption, heavy metals, by-products, pollution, agroindustry. 


\section{INTRODUCCIÓN}

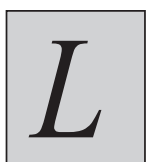

a Agencia de Protección Ambiental de los Estados Unidos (USEPA, por sus siglas en inglés), establece cinco metales pesados como los más relevantes en cuanto a su impacto a la salud; Cadmio, Cromo, Cobre, Plomo y Mercurio (USEPA, 2007). La fuente principal de contaminación de metales pesados son las actividades provocadas por el hombre, que involucra: acabado de metales, rellenos sanitarios, actividades agrícolas, actividades industriales (minería, textil, galvanoplastia), producción de energía y procesamiento de cuero, entre otras (Akpor, Ohiobor \& Olaolu, 2014; Covarrubias \& Peña Cabriales, 2017; Acosta, López, Coronado, Cárdenas \& Martínez, 2010; Bankar \& Nagaraja, 2018; Sardar et al., 2013).

La comunidad científica se ha esforzado en desarrollar métodos y procesos para la remoción de metales pesados en ambientes contaminados, generalmente efluentes industriales; los procesos son: adsorción (Crini, 2005; Gautam, Mudhoo, Lofrano \& Chattopadhyaya, 2014), precipitación, intercambio iónico, electrodeposición (Kurniawan, Chan, Lo \& Babel, 2006), coagulación-floculación (Aderhold, Williams \& Edyvean, 1996), flotación (Rubio, Souza \& Smith, 2002) y filtración con membranas (O'Connell, Birkinshaw \& O'Dwyer, 2008). La principal desventaja de estos métodos es su alto costo de inversión, operación y mantenimiento, además algunos generan lodos residuales, por lo que son un problema ambiental (Rangabhashiyam \& Balasubramanian, 2019).

Uno de los métodos para la remoción de metales pesados es la bioadsorción, con base en un proceso fisico-químico al que está involucrado un material biológico (e. g. biomasa viva o muerta) como adsorbente y una fase líquida (efluente acuoso) que contiene disuelta la sustancia problema (iones de metales pesados) (Volesky, 2007; Gadd, 2008; Tejada Tovar, Villabona Ortiz \& Garcés Jaraba, 2015). En comparación con los métodos mencionados anteriormente, la bioadsorción presenta una alta eficiencia de remoción, regeneración del bioadsorbente y es libre de formación de contaminantes secundarios (lodos contaminados) (Rangabhashiyam \& Balasubramanian, 2019; Santos, Ungureanu, Volf, Boaventura \& Botelho, 2018; Bankar \& Nagaraja, 2018; Ahmet, Özgur \& Tuzen 2011; Rangabhashiyam, Selvaraju, Selvaraju \& Varguese, 2014). Para la bioadsorción es viable utilizar biomasa muerta de diversas fuentes biológicas con la ventaja que no requiere de medios de crecimiento (cultivos), es de fácil desorción y están disponibles como residuos o subproductos agroindustriales, lo cual proporciona la ventaja de utilizar subproductos como un bioadsorbente de bajo costo con aplicación para aguas contaminadas e. g. aguas residuales industriales (Gupta, Rastogi \& Nayak, 2010; Malik, 2004; Rangabhashiyam, Lata \& Balasubramanian, 2018; Dhankhar \& Hooda, 2011; Baysal,
Cinar, Bulut, Alkan \& Dogru, 2009; Farooq, Kozinski, Khan \& Athar, 2010).

La interacción entre el bioadsorbente (subproductos agroindustriales) con los iones de metales pesados es compleja, ya que es posible que intervengan tres mecanismos: complejación (Sag \& Kursal, 2001; Naja \& Volesky, 2011; Diniz, Weber, Volesky \& Naja, 2008), micro-precipitación (Ahalya, Kanamadi \& Ramachandra, 2006; Mclean, Fortin \& Brown, 2011; Naja \& Volesky, 2011) e intercambio iónico (Uluozlu, Sari, Tuzen \& Soylak, 2008; Naja, Murphy \& Volesky, 2010; Svecova, Spanelova, Kubal \& Guibal, 2006; Won, Han \& Yun, 2008; Sutherland \& Venkobachar, 2010). Estos mecanismos ocurren debido a los grupos funcionales que se encuentran en la superficie y poros del bioadsorbente como son: hidroxilo, carboxilo, amino, tiol, entre otros (Volesky, 2007; Javanbakht, Alavi Amir \& Zilouei, 2014). La bioadsorción está ligada a propiedades del bioadsorbente, así como a características del efluente a tratar, debido a esto el proceso puede presentar cambios negativos o positivos por lo que es dependiente de los parámetros de operación como: pH (Naja \& Volesky, 2011; Kuyucak \& Volesky, 1989; Greene, Henzl, Hosea \& Darnall, 1986), concentración del metal pesado en el efluente (Enniya, Rghioui \& Jourani, 2018), cantidad de bioadsorbente utilizado (Enniya et al., 2018; Anwar et al., 2010), temperatura (Naja \& Volesky, 2011) y presencia de otros metales (Mendoza-Castillo, 2012), por lo que es necesario realizar estudios para obtener los parámetros de operación de la bioadsorción empleando un bioadsorbente específico.

En México, la agroindustria es una de las actividades más importantes debido a su crecimiento en los últimos años (Agro Revista Industrial del Campo, 2016) y es la que más genera subproductos que no son aprovechados (Valdez, Acevedo \& Hernández, 2010), entre ellos se encuentran: el bagazo de café, el agave, maguey, caña de azúcar, pajas de distintos cultivos, residuos orgánicos de frutas y verduras (González S., Pérez F., Wong W., Bello M., \& Yáñez 0., 2015). Por lo anterior, el objetivo de esta revisión, es contribuir con información sistematizada para brindar un panorama del método de remoción de metales por bioadsorción, a través del uso de subproductos agroindustriales para la descontaminación de efluentes contaminados con metales pesados.

\section{Metales Pesados}

Metales pesados es un término general aplicado al grupo de metales y metaloides, que en su mayoría son tóxicos para los seres humanos, incluso a baja concentración (Vardhan, Kumar \& Panda, 2019; Bankar \& Nagaraja, 2018). La USEPA (2007) toma en cuenta cinco metales pesados como los más relevantes en cuanto a su impacto a la salud, que se muestran en la Tabla I , se detalla la concentración máxima permisible en cuerpos de agua de acuerdo a distintas organizaciones, así como el 
Tabla I. Concentración máxima permisible en cuerpos de agua y daños a la salud.

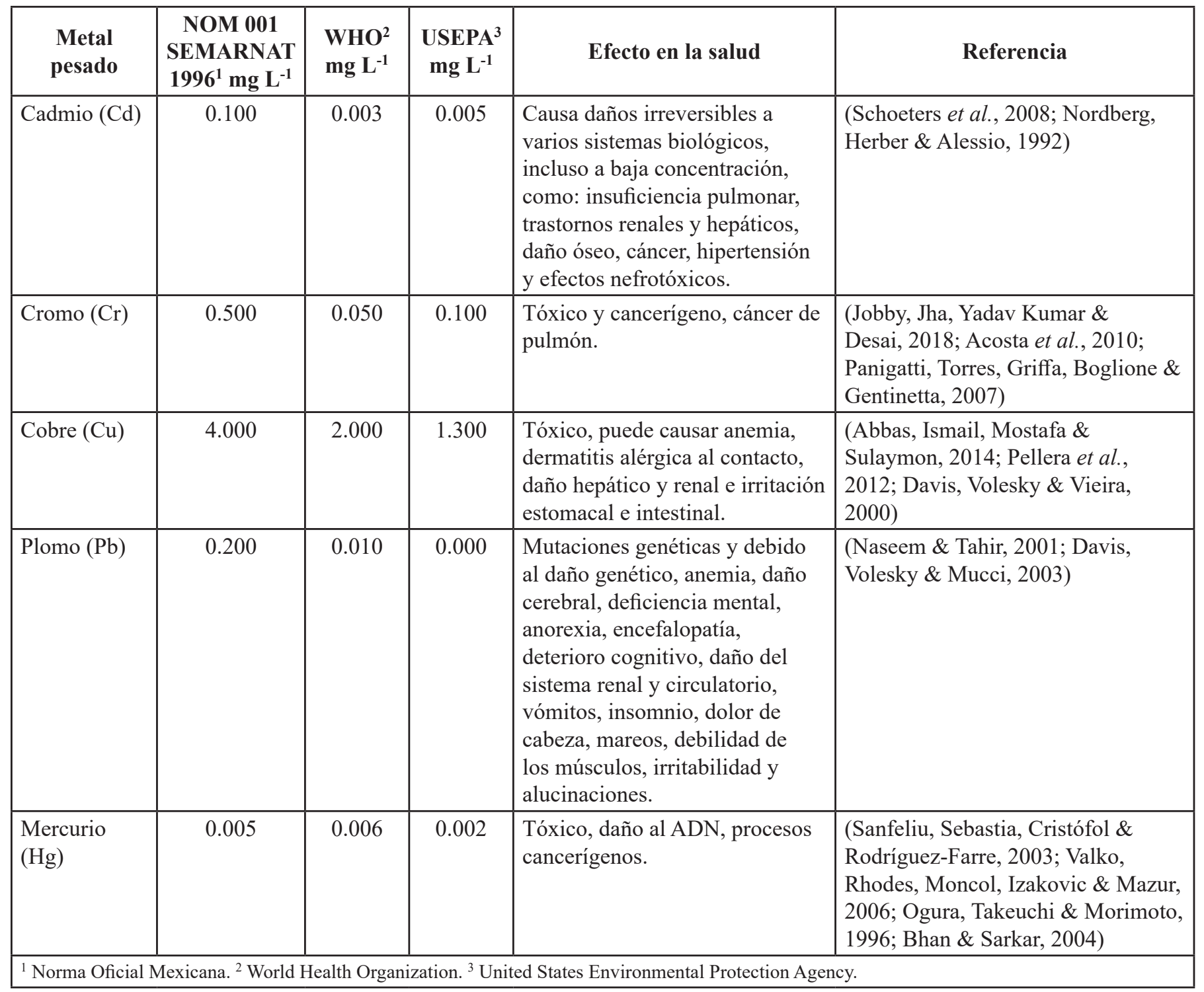

daño a la salud por la presencia de estos metales en el cuerpo humano, razón imprescindible para encontrar alternativas $\mathbf{y}$ evitar la contaminación o en su caso la remoción de metales en los cuerpos de agua.

Existen dos fuentes principales de metales pesados, la natural y antropogénica; la primera incluye intemperismo de rocas (Kaizer \& Osakwe, 2010) y actividad volcánica (Amaral, Cruz, Cunha \& Rodrigues, 2006) mientras que la fuente antropogénica son acciones provocadas por el hombre, que se pueden englobar en cinco categorías: las actividades industriales, agrícolas (pesticidas y herbicidas), disposición de residuos, minería y metalurgia. La contaminación ocasionada por los residuos derivados de éstas, se definirá por la estabilidad química y la solubilidad de los metales pesados de las que dependerá una amplia variedad de impactos en la salud humana y el ambiente (Akpor et al., 2014; Covarrubias \& Peña Cabriales, 2017; Acosta et al., 2010; Bankar \& Nagaraja, 2018; Burakov et al., 2018; Sardar et al., 2013). Es importante destacar que la contaminación por metales pesados es uno de los problemas más graves en los entornos marinos, terrestres y de agua dulce (Valdman, Erijman, Pessoa \& Leite, 2001; Ansari \& Malik, 2007).

\section{MÉTOdOS FÍSICO-QUÍMICOS DE REMOCIÓN DE METALES PESADOS}

Los metales pesados son compuestos requeridos en distintas industrias y son necesarios para la economía y crecimiento de cualquier país (Dursun, 2006). Sin embargo, el uso irracional de ellos, ha llevado a la comunidad científica a desarrollar 
métodos para la remoción de metales pesados en ambientes contaminados o en efluentes industriales, englobando los siguientes procesos:

\section{Precipitación}

La precipitación emplea aniones para precipitar iones metálicos insolubles en agua de manera eficiente $\mathbf{y}$ a concentraciones superiores a $1000 \mathrm{mg} \mathrm{L}^{-1}$, principalmente el precipitado está en forma de hidróxidos, sulfatos, carbonatos, sulfuros y fosfatos. Sin embargo, factores como el $\mathrm{pH}$ y la presencia de otros iones afectan la eficiencia del proceso, además es importante señalar que implica el uso de precipitantes químicos adicionales, generando lodos y un riesgo ambiental mayor (Kurniawan et al., 2006).

\section{Intercambio iónico}

Este proceso hace uso de intercambiadores de iones de forma sólida, generalmente son resinas con sitios activos como: $\mathrm{SO}_{3}$, $\mathrm{COO}^{-}, \mathrm{N}^{+}\left(\mathrm{CH}_{3}\right)_{3}$ y $\mathrm{N}^{+} \mathrm{H}_{3}$, que intercambian aniones o cationes con la solución problema. El proceso presenta una cinética de remoción rápida, gran capacidad de tratamiento y eficiente a baja concentración de metales pesados. Sin embargo, los intercambiadores de iones son costosos y altamente sensibles al pH y a sólidos suspendidos (Kurniawan et al., 2006; Abdullah, Yusof, Lau, Jaafar \& Ismail, 2019).

\section{Electrodeposición}

Este proceso implica el paso de una corriente eléctrica a través de un metal con soporte acuoso, que consiste en una placa de cátodo y un ánodo. Los iones metálicos positivamente se adhieren como precipitados a la superficie de la carga negativa (cátodo) formando una capa como se muestra en la Figura 1, este proceso conduce a la deposición selectiva del metal, Gu et al., (2020) estudiaron la recuperación del cobre mediante electrodeposición de agua residual obteniendo una recuperación del $89.3 \%$. Las desventajas de este proceso es la corrosión y la frecuencia de reemplazo de los electrodos (Kurniawan et al., 2006).

\section{Coagulación-Floculación}

La coagulación-floculación remueve el metal pesado en la solución acuosa a concentraciones altas, mediante polímeros que forman un puente entre flóculos y los iones metálicos obteniendo grandes aglomerados. La ventaja de este proceso

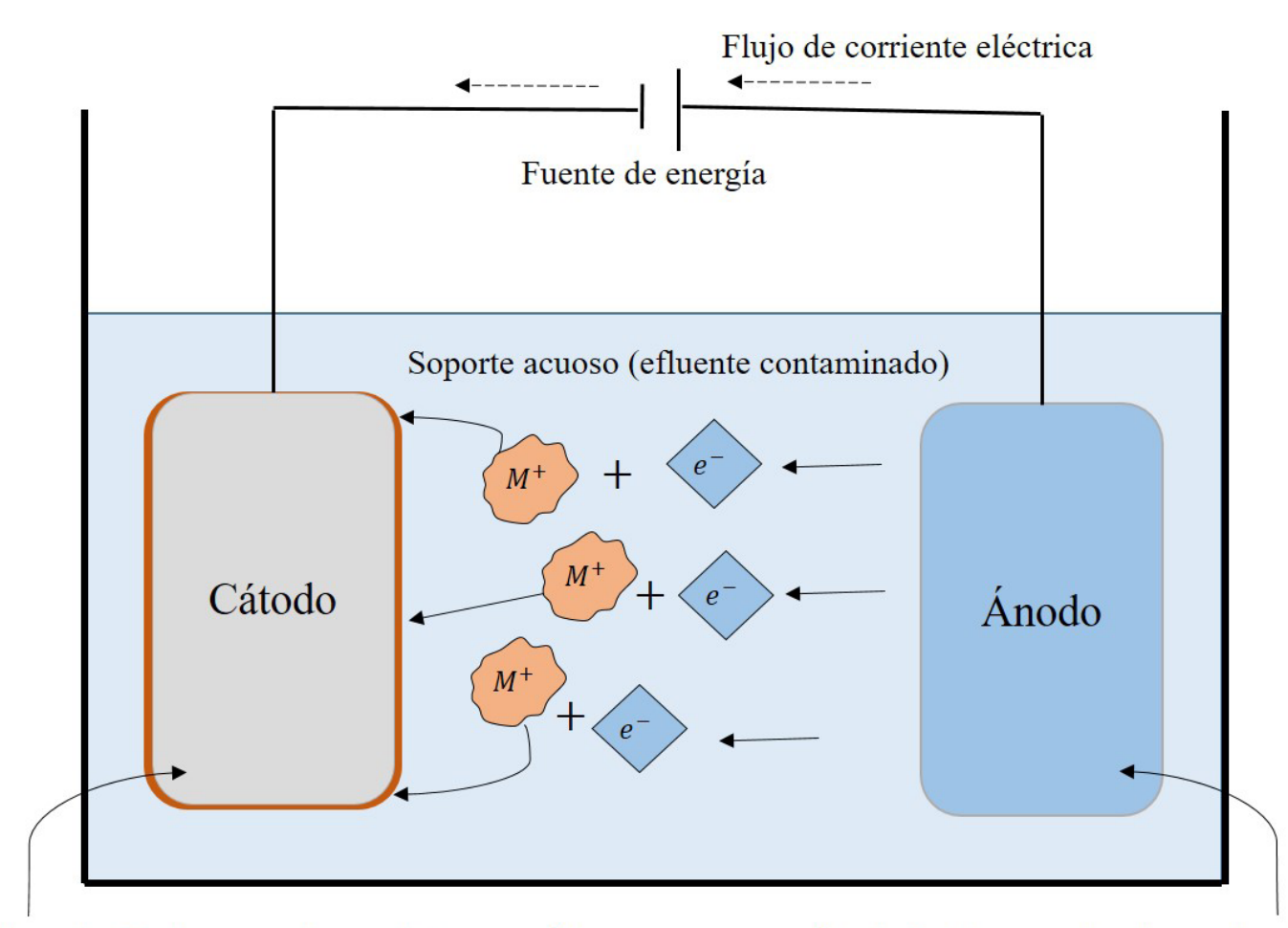

En el cátodo se reduce el ion metálico, formando precipitados adheridos al cátodo:

$$
M^{n+}+n e^{-} \rightarrow M^{0}
$$

En el ánodo se realiza la producción de oxígeno y electrones:

$$
\mathrm{H}_{2} \mathrm{O} \rightarrow 0.5 \mathrm{O}_{2} \uparrow+2 \mathrm{H}^{+}+2 e^{-}
$$


es el buen asentamiento de los lodos y su facilidad de deshidratación, mientras que las desventajas son: la presencia de sólidos suspendidos que afectan la eficiencia del proceso, se consume una gran cantidad de químicos y genera gran cantidad de lodos residuales (Aderhold et al., 1996; Abdullah et al., 2019).

\section{Flotación}

La flotación involucra burbujas de aire para separar metales pesados de una solución acuosa. Los méritos de este proceso incluyen un bajo tiempo de retención y la remoción de pequeñas partículas, así como sólidos suspendidos presentes en la solución. El inconveniente de este método es el alto costo de inversión inicial y mantenimiento, además, se requiere de efluentes con concentraciones muy altas del metal pesado (Rubio et al., 2002).

\section{Filtración por membranas}

Los procesos de filtración con membranas presentan una menor generación de desechos sólidos, un consumo mínimo de químicos, menor espacio de trabajo y remoción selectiva de metales; sin embargo, se necesita un alto costo de inversión inicial, de mantenimiento y operación. Además, el proceso durante un periodo de tiempo determinado provoca taponamientos de la membrana que afecta directamente el caudal del tratamiento y su eficiencia (O'Connell et al., 2008).

\section{Adsorción}

La adsorción es un método efectivo para la remoción de metales pesados en aguas residuales industriales, ofrece ventajas de flexibilidad de diseño y regeneración eficiente del adsorbente por desorción. El rendimiento del proceso depende del tipo de adsorbente utilizado, generalmente es carbón activado, sin embargo, es importante destacar que es un material costoso (Crini, 2005; Gautam et al., 2014). Los métodos físico-químicos de remoción de metales pesados como se mencionó, no son económicamente viables por sus altos costos de inversión, operación y mantenimiento (Kurniawan et al., 2006; Eccles, 1999). Además de la generación de lodos químicos, éstos presentan un problema ambiental mayor (Rangabhashiyam \& Balasubramanian, 2019).

En los últimos años se ha buscado un método alternativo para combatir los problemas asociados con la remoción de metales pesados en medios acuosos, se ha estudiado la utilización de biomasa como medio adsorbente, desarrollando así, la bioadsorción como método de remoción de metales pesados, siendo uno de los métodos importantes en esta revisión.

El término bioadsorción se refiere a un proceso físicoquímico que ocurre entre dos sustancias, en especial la unión de una especie química a una superficie. El término "bio" involucra el uso de material de origen biológico, e. g. biomasa (viva o muerta) para adsorber o concentrar iones $\mathrm{u}$ otras moléculas presentes en la superficie de una solución acuosa (Volesky, 2007; Gadd, 2008). El proceso de bioadsorción involucra una fase sólida (bioadsorbente) y una fase líquida (agua) que contiene disuelta la sustancia de interés (iones de metales pesados) (Tejada Tovar et al., 2015). En la práctica, la bioadsorción presenta ventajas como bajo costo de operación, es amigable con el ambiente, reutilización del bioadsorbente, tiempo de operación corto, alta eficiencia y libre de formación de contaminantes secundarios (lodos) (Rangabhashiyam \& Balasubramanian, 2019; Santos et al., 2018; Bankar \& Nagaraja, 2018; Ahmet et al., 2011; Rangabhashiyam et al., 2014).

Existen dos métodos de operación de la bioadsorción: (1) batch, que consiste en agregar el bioadsorbente en contacto con una solución acuosa que contiene los iones metálicos hasta que se alcance el equilibrio, posteriormente, el bioadsorbente se separa de la fase líquida y se regenera o se dispone de manera ambientalmente aceptable. (2) Continua, que consiste en una columna empacada o de lecho fluidizado en la que el bioadsorbente una vez cargado en la columna se pone en contacto con una alimentación continua de solución acuosa con los iones metálicos, de igual manera una vez que se satura el bioadsorbente, se regenera o se dispone de manera aceptable (Aksu, 2005).

Para el proceso de bioadsorción se prefiere utilizar biomasa muerta de varias fuentes biológicas, sobre la viva, ya que la biomasa muerta no requiere medios de crecimiento, y como bioadsorbente generalmente no se ve afectada por la toxicidad de los metales pesados. El uso de la biomasa ofrece los beneficios de un tamaño de bioadsorbente uniforme, regeneración simple, remoción efectiva a baja concentración de metal y ajuste a modelos matemáticos que describen como se lleva a cabo la bioadsorción (Gupta et al., 2010; Malik, 2004; Rangabhashiyam et al., 2018; Dhankhar \& Hooda, 2011; Baysal et al., 2009).

\section{Mecanismos de bioadsorción}

La interacción de los iones de metales pesados y el bioadsorbente es un proceso complejo en donde es posible que intervengan tres de los mecanismos siguientes (Rangabhashiyam \& Balasubramanian, 2019; Khosa \& Ullah, 2018; Javanbakht et al., 2014; Michalak, Chojnacka \& Witek Krowiak, 2013; Montazer-Rahmati, Rabbani, Abdolali \& Keshtkar, 2011).

\section{Complejación}

La complejación se define como la formación de una macromolécula mediante la asociación de dos o más especies, cuando una de las especies es un ion metálico. La remoción del metal pesado en solución puede tener lugar mediante una formación compleja en la superficie del bioadsorbente después de la interacción entre el metal y grupos activos como 
el carboxilo, amino, tiol, hidroxilo, fosfato e hidroxicarboxilo de la superficie del bioadsorbente que pueden interactuar de manera coordinada con iones de metales pesados (Sag \& Kursal, 2001; Naja \& Volesky, 2011). La complejación juega un papel importante en las interacciones metalligando y sorbato-adsorbente, especialmente en la biomasa lignocelulosica (Diniz et al., 2008).

Ahalya et al., (2006) encontraron que ácidos orgánicos como: (e. g. el ácido cítrico, oxálico, gluónico, fumárico, láctico y málico) son capaces de formar quelatos con metales pesados formando moléculas metalorgánicas. La unión de diferentes grupos funcionales depende de las propiedades iónicas como la electronegatividad, el potencial de ionización, el radio iónico y el potencial redox de los metales (Allard, Hakansson \& Karlsson, 2005).

\section{Micro-Precipitación}

La micro - precipitación es el resultado entre la reacción de los iones metálicos y los compuestos que se encuentran en la superficie (Ahalya et al., 2006). Es a menudo precedido por la unión a sitios específicos que proporcionan puntos de nucleación ${ }^{1}$ (Mclean et al., 2011).

Las condiciones para que se lleve a cabo este mecanismo pueden ser creadas por desviaciones locales en condiciones físicas como el $\mathrm{pH}$ o por la presencia de materiales del propio bioadsorbente, en el proceso de bioadsorción, la microprecipitación puede contribuir a la eficiencia de remoción del metal, dado que el micro-precipitado es recogido por la fase sólida y así inmovilizado y separado de la solución (Naja \& Volesky, 2011).

\section{Intercambio iónico}

El término intercambio iónico no identifica explícitamente el mecanismo de unión de metales pesados a la biomasa, ya que los mecanismos precisos pueden variar desde la unión física (es decir, fuerzas electrostáticas o de London-Van der Waals) hasta la unión química (es decir, iónica o covalente) (Uluozlu et al., 2008; Won et al., 2008; Sutherland \& Venkobachar, 2010).

La atracción electrostática en la bioadsorción es dependiente de los tipos y cantidad de sitios activos presentes en la biomasa, y si estos están ionizados u ocupados por protones y otros iones. Eso, a su vez, depende del $\mathrm{pH}$ y el $\mathrm{pKa}^{2}$ del grupo funcional respectivo, e. g. los grupos amino están cargados positivamente, los grupos carboxilo, sulfato y fosfato tienen

\footnotetext{
1 Punto de formación de precipitados por aglomeración.
}

2 La constante pKa es una medida de la acidez de una sustancia a partir de su constante de disociación ácida. El valor de $\mathrm{pKa}$ indica que a ese valor de $\mathrm{pH} 50 \%$ de la especie esta disociada y el otro $50 \%$ se encuentra asociada. carga negativa cuando estos están desprotonados (Naja et al., 2010). Un aumento en el pH de la solución del ion metálico durante la bioadsorción hace que los protones se liberen de la superficie, creando así sitios de intercambio iónico (Svecova et al., 2006).

Estos mecanismos presentes en la bioadsorción como se estableció anteriormente, son posibles debido a grupos funcionales que se encuentran en la superficie y en poros del bioadsorbente, en la Figura 2 se esquematizan los mecanismos detallados anteriormente, además en la Tabla II se muestran los grupos funcionales involucrados en la bioadsorción, que suelen ser afectados por características físico-químicas de la solución problema o parámetros de operación del método (Volesky, 2007; Javanbakht et al., 2014).

\section{SUBPRODUCTOS AGROINDUSTRIALES COMO MATERIAL BIOADSORBENTE}

Se han examinado una gran variedad de adsorbentes de bajo costo por su capacidad para remover distintos contaminantes en medios acuosos y en aguas residuales industriales. De manera general, diversos estudios han buscado alternativas para competir con el carbón activado como agente adsorbente, específicamente buscando materiales accesibles y de fácil preparación. Una de las alternativas exploradas ha sido el uso de subproductos agroindustriales generados por la agroindustria.

La agroindustria es una actividad que integra la producción primaria agrícola, pecuaria $\mathrm{y}$ forestal, el proceso de transformación, así como la comercialización del producto. Es decir, una actividad económica que combina el proceso productivo agrícola con el industrial para generar alimentos o materias primas semi-elaboradas destinadas al mercado (Cury R., Aguas M., Martínez M., Olivero R. \& Chamus Ch., 2017).

La agroindustria genera residuos o subproductos de bajo costo y fácilmente disponibles como: el bagazo del agave (Velázquez Jiménez, Pavlick \& Rangel Méndez, 2013), la cáscara del plátano (Anwar et al., 2010), la paja de cebada (Pehlivan, Altun \& Parlayici, 2012) y las cáscaras de: la mandarina (Husein, 2013), el mango (Iqbal, Saeed \& Kalim, 2009), el melón (Huang \& Zhu, 2013), la lima (Saha, Mukherjee \& Saha, 2013), la toronja (Tasaso, 2014), y el arroz (Aydin et al., 2008), la paja de avena (Aydin, Bulut \& Yerlikaya, 2008), las cáscaras de: coco (Sousa et al., 2010), y naranja (Sha \& Ningchuan, 2009), mazorca de maíz (Tan, Yuan, Liu \& Xiao, 2010), y el bagazo del azúcar (Alomá, Martín-Lara, Rodríguez, Blázquez \& Calero, 2012), entre otros.

En México, los subproductos agroindustriales se obtienen de cultivos que incluyen el maíz, trigo, sorgo, frijol, arroz, soya, caña, cebada y cacahuate, mientras que dentro de los 


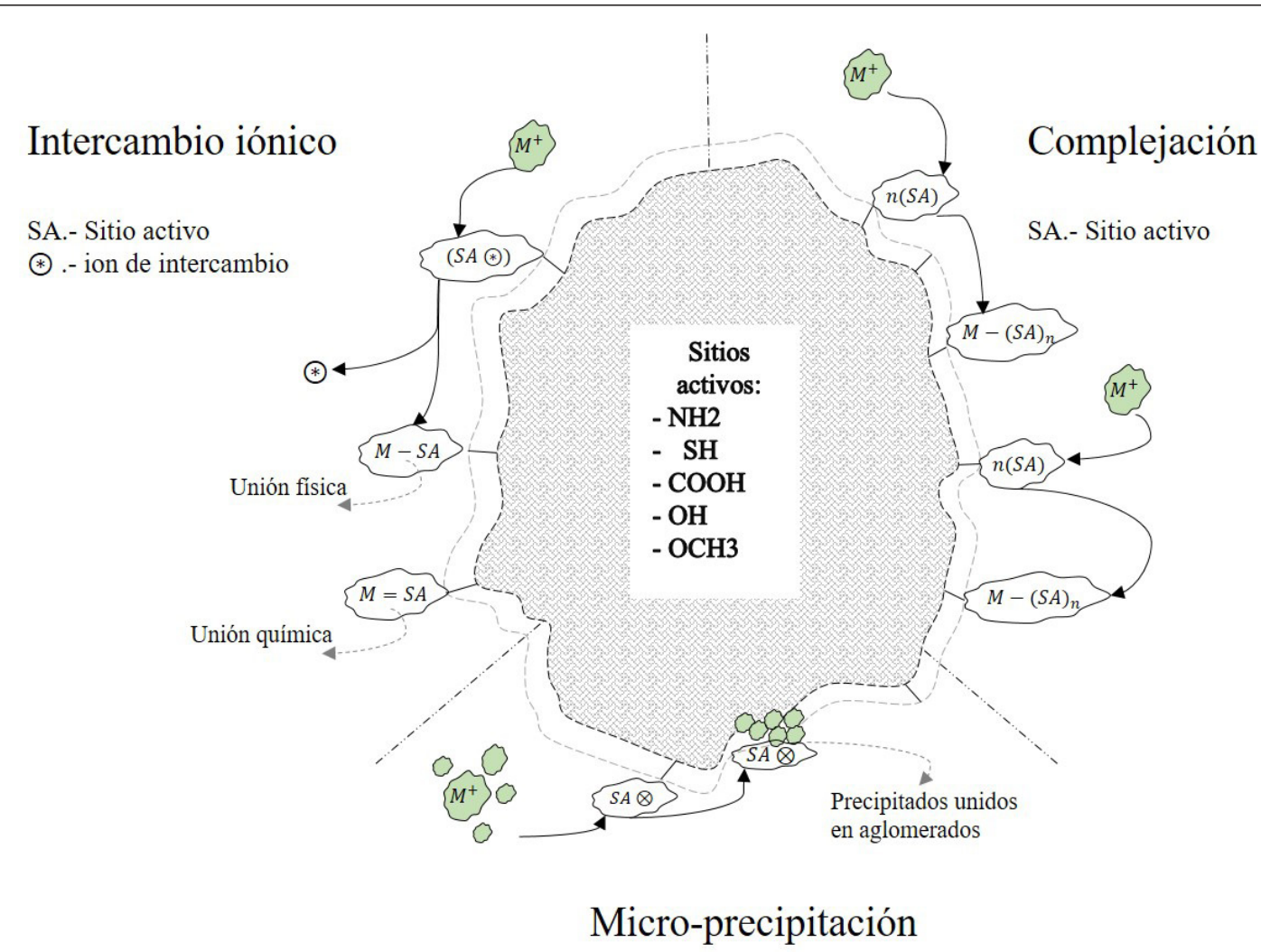

SA.- Sitio activo

$\otimes$.- Punto de nucleación

Figura 2. Mecanismos involucrados en la bioadsorción utilizando subproductos agroindustriales $\left(\mathrm{M}^{+}\right.$, ion metálico). Elaboración personal.

Tabla II. Grupos funcionales involucrados en la bioadsorción.

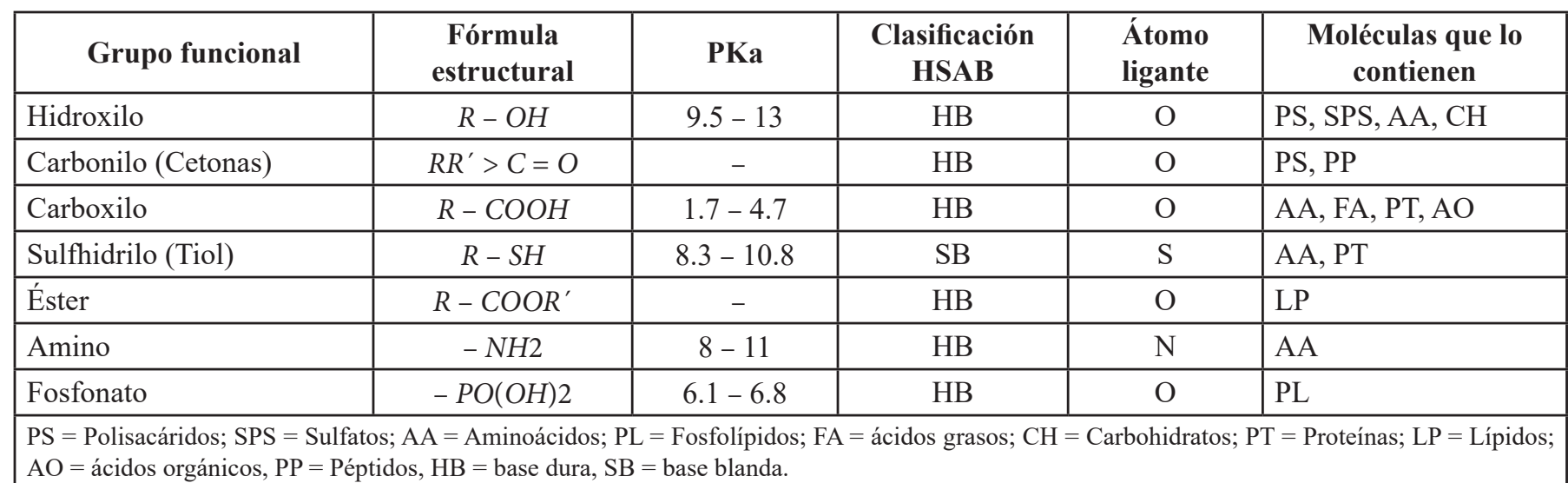

(Volesky, 2007; Javanbakht et al., 2014; Alfarra, Frackowiak \& Béguin, 2004). 
subproductos secundarios (los bagazos, tallos, semillas y paja) están también el algodón, cártamo, agave, maguey y café (Casas \& Sandoval, 2014).

Las características o composición química y biológica de los subproductos agroindustriales dependen del proceso de transformación y de la materia prima utilizada. Sin embargo, los componentes básicos de los subproductos incluyen hemicelulosa, lignina, lípidos, proteínas, azúcares simples y almidón, que contienen una variedad de grupos funcionales con capacidad de adsorción potencial para metales pesados (Casas \& Sandoval, 2014; Bhatnagar \& Sillanpaa, 2010; Bhatnagar, Sillanpaa \& Witek-Krowiak, 2015).

Los subproductos se utilizan en la bioadsorción de forma natural y modificada, en la forma natural, el material se lava, se tritura y se tamiza hasta alcanzar el tamaño de partícula deseado y posteriormente se utiliza en pruebas de bioadsorción batch o continua. En la forma modificada, el material se trata previamente mediante técnicas de modificación, generalmente se deja en reposo en soluciones ácidas o básicas, con el objetivo de mejorar y reforzar el potencial del grupo funcional con los que posee y en consecuencia, aumentar el número de sitios activos para adsorber metales pesados (Bhatnagar \& Sillanpaa, 2010).

La importancia de utilizar subproductos agroindustriales no sólo radica en su capacidad de adsorción, sino en la facilidad de su regeneración para su reutilización. La regeneración involucra diferentes agentes o mecanismos para modificar el equilibrio de adsorción hacia la desorción del ion metálico, que se clasifican en tres grupos: regeneración térmica, química y microbiológica (Salvador, Martín-Sánchez, SánchezHernández, Sánchez-Montero \& Izquierdo, 2015a; Salvador, Martín-Sánchez, Sánchez-Hernández, Sánchez-Montero \& Izquierdo, 2015b).

En general, la regeneración química es el enfoque más utilizado para reactivar los adsorbentes utilizando compuestos químicos como $\mathrm{HCl}, \mathrm{HNO}_{3}, \mathrm{NaOH}$, EDTA, $\mathrm{CaCl}_{2} \mathrm{y} \mathrm{Na}_{2} \mathrm{CO}_{3}$, etc. En muchos casos, los ácidos o álcalis minerales diluidos permiten una desorción eficiente; sin embargo, el uso de estas sustancias puede causar daños en el bioadsorbente ocasionando una disminución en la capacidad de adsorción después de la regeneración (Vijayaraghavan \& Yun, 2008; Park, Yun, \& Park, 2010; Nijkam \& Schiewer, 2012). Una vez que el bioadsorbente llega a su saturación se procede a su disposición final como residuo peligroso o se puede llevar a cabo la pirólisis, que mayormente se utiliza para disminuir la cantidad de material adsorbente saturado a cenizas que se llevan posteriormente a disposición final (Liu et al., 2020).

La desorción también permite la recuperación de los contaminantes adsorbidos que pueden clasificarse como productos químicos peligrosos y llevarlos a disposición final o reintegrarlos a los procesos productivos (BonillaPetriciolet, Mendoza-Castillo \& Reynel, 2017). En general, los subproductos representan un reto importante respecto a su disposición final, esto debido a la capacidad que presentan para generarse y sus efectos en el medio ambiente (Gisi, Lofrano, Grassi \& Notarnicola, 2016). Si estos residuos se utilizaran como adsorbentes de bajo costo, proporcionarían la ventaja de utilizar los subproductos como un bioadsorbente con aplicación para aguas contaminadas dándole así valor agregado (Grassi, Kaykioglu, Belgiorno \& Lofrano, 2012).

\section{Factores que influyen en la eficiencia de la bioadsorción}

La eficiencia de la bioadsorción del metal tóxico está ligada a propiedades del bioadsorbente, así como a parámetros de operación como: temperatura, $\mathrm{pH}$, tamaño del bioadsorbente, presencia de otros iones, tiempo de contacto y concentración del metal pesado contaminante (Naja \& Volesky, 2011; Hlihor, Bulgariu, Sobariu, Diaconu, Tavarea \& Gavrilescu, 2014; Rao \& Prabhakar, 2011; Rao, Mohapatra, Anand \& Venkateswarlu, 2010). A continuación, se detallan algunos de los parámetros más relevantes del proceso de bioadsorción.

\section{pH}

Hay tres maneras en el que el $\mathrm{pH}$ puede influir en la bioadsorción de los metales:

(1) El pH de la solución podría cambiar el estado de los sitios químicamente activos cuando los grupos de unión a metales son débilmente ácidos o básicos, la disponibilidad de sitios libres depende del pH (Naja \& Volesky, 2011).

(2) A valores extremos de $\mathrm{pH}$ utilizados para la regeneración (desorción) pueden dañar la estructura del material bioadsorbente, generalmente se observa una pérdida de biomasa y disminución de la capacidad de adsorción (Kuyucak \& Volesky, 1989).

(3) La especiación del metal en solución depende del $\mathrm{pH}$, esto quiere decir que cuando el $\mathrm{pH}$ es bajo se producen iones metálicos de alta carga y tamaño pequeño, a $\mathrm{pH}$ básicos la presencia de grupos hidroxilo puede generar la formación de complejos disminuyendo la eficiencia de remoción. Por lo que a $\mathrm{pH}$ ácido los grupos funcionales competirán por sitios de unión activa con iones metálicos, aumentando su remoción (Greene et al., 1986).

\section{Concentración de metal pesado.}

La eficiencia del adsorbente aumenta a medida que aumenta la concentración del metal hasta una concentración constante en la que comienza a disminuir, esto se atribuye al hecho de que la superficie disponible de adsorbente se vuelve más pequeña 
a medida que los iones metálicos se adsorben en la superficie del bioadsorbente. Cuando la concentración del metal es suficientemente grande, la superficie del adsorbente se satura, la eficiencia comienza a disminuir y los iones metálicos se pueden llegar a desorber (Enniya et al., 2018).

\section{Cantidad del bioadsorbente}

La bioadsorción tiene lugar fundamentalmente en poros del bioadsorbente o sobre la superficie de estos en puntos específicos. La cantidad de adsorbato (metal pesado) que se puede adsorber es directamente proporcional al volumen del adsorbente, y este volumen es directamente proporcional al área externa con una partícula pequeña que tiene mayor área superficial. Entre mayor sea el área de superficie externa se incrementará la cantidad de sitios activos para la bioadsorción (Enniya et al., 2018; Anwar et al., 2010).

\section{Temperatura}

El efecto de la temperatura sobre la bioadsorción depende del calor de adsorción (entalpía de cambio), para la adsorción física el calor de la entalpía es $<0$, la reacción de adsorción es exotérmica y se prefiere a temperaturas bajas, para adsorción química el valor de la entalpía es $>0$, por lo que la reacción de adsorción es endotérmica y se favorece a temperaturas altas (Naja \& Volesky, 2011).

\section{Presencia de otros metales}

La presencia de iones en la solución problema hace que estos puedan competir con el metal por zonas de adsorción. Por otra parte, Mendoza Castillo (2012) estudió la sorción multicomponente $\mathrm{Cd}^{+2}, \mathrm{Ni}^{+2}$ y $\mathrm{Zn}^{+2}$ en solución acuosa, los resultados obtenidos indican que la capacidad de sorción es inferior a la obtenida en los sistemas monometálicos, y, por lo tanto, se puede concluir que existe un efecto de competencia en el proceso de sorción entre los metales evaluados, es importante destacar que se requieren más estudios de bioadsorción multicomponente.

\section{Composición química del bioadsorbente}

Los componentes básicos de un subproducto agroindustrial son polisacáridos estructurales como la celulosa (30-50\%), hemicelulosa (20-40\%) y lignina (15-25\%). La composición química de algunos materiales lignocelulósicos comunes se presentan en la Tabla III. El tipo de grupos funcionales y componentes químicos en los subproductos lignocelulósicos son similares, pero en cantidades diferentes, por lo que desempeñan un papel importante en la adsorción de los iones metálicos (Asadi, Shariatmadari \& mirghaffari, 2008).

Un componente clave en la bioadsorción es la lignina, ya que es una parte integral de la matriz lignocelulósica que le confiere resistencia mecánica, para su utilización como material adsorbente, además, la lignina contiene una gran variedad de grupos funcionales como: grupos hidroxilo alifáticos y fenólicos, grupos metoxilo y grupo carbonilo, que pueden tener la capacidad de unirse a iones metálicos mediante la donación del par de electrones del oxígeno y formar complejos estables (Miretzky \& Fernández Cirelli, 2010; Fiol et al., 2006 ). En la Tabla IV, se presentan los valores de la capacidad máxima de bioadsorción para diversos subproductos utilizados de manera natural.

\section{PotenCIAL DE SUBPRODUCTOS AGROINDUSTRIALES COMO BIOADSORBENTES EN MÉXICO}

Existen factores clave para un desarrollo sostenible eficaz, eficiente y son: la demanda energética, el cambio climático, la escasez de recursos naturales y el manejo de los residuos. Bajo este contexto, la agroindustria mexicana, sus cadenas de producción y servicios, generan un gran número de residuos que no son aprovechados (Valdez V. et al., 2010).

Tabla III. Composición química de la biomasa lignocelulósica.

\begin{tabular}{|l|c|c|c|c|c|}
\hline \multirow{2}{*}{ Biomasa } & \multicolumn{5}{c|}{ Composición química (\%) } \\
\cline { 2 - 6 } & Celulosa & Lignina & Hemicelulosa & Ceniza & Sílica \\
\hline Paja de arroz & $25-35$ & $10-15$ & $20-30$ & $15-20$ & $9-15$ \\
\hline Paja de avena & $30-35$ & $16-21$ & $26-32$ & $4.5-9$ & $3-7$ \\
\hline Paja de cebada & $30-35$ & $14-15$ & $24-29$ & $5-7$ & $3-6$ \\
\hline Bagazo de caña de azúcar & $32-44$ & $19-24$ & $25-35$ & $1.5-5$ & $<4$ \\
\hline Bamboo & $26-43$ & $21-31$ & $15-26$ & $1.7-5$ & $<1$ \\
\hline Maíz & $35-45$ & $5-15$ & $35-45$ & $1-2$ & $<1$ \\
\hline Residuos de algodón & $80-95$ & - & $5-20$ & $<1$ & - \\
\hline Madera & $40-50$ & $25-35$ & $25-35$ & $<1$ & - \\
\hline Hueso de olivo & $30-35$ & $20-25$ & $20-30$ & $<1$ & $5-9$ \\
\hline Cáscara de nuez & $25-35$ & $30-40$ & $25-30$ & - & - \\
\hline \multicolumn{7}{|l|}{} \\
\hline
\end{tabular}


Tabla IV. Capacidad máxima de bioadsorción para distintos subproductos agroindustriales.

\begin{tabular}{|c|c|c|c|}
\hline Biomasa & Adsorbato & $\begin{array}{l}\text { Capacidad máxima de } \\
\text { adsorción (mg/g) }\end{array}$ & Referencia \\
\hline Bagazo de agave & $\mathrm{Cd}(\mathrm{II})$ & 13.27 & (Velázquez et al., 2013) \\
\hline Cáscara de kiwi & $\mathrm{Cd}(\mathrm{II})$ & 470 & (Al-Qahtani, 2016) \\
\hline Cáscara de mandarina & $\mathrm{Cd}(\mathrm{II})$ & 450 & (Al-Qahtani, 2016) \\
\hline Cáscara de plátano & $\mathrm{Cd}(\mathrm{II})$ & 195 & (Al-Qahtani, 2016) \\
\hline Cáscara de plátano & $\mathrm{Cd}(\mathrm{II})$ & 5.71 & (Anwar et al., 2010) \\
\hline Vástago de trigo & $\mathrm{Cd}(\mathrm{II})$ & 11.6 & (Tan \& Xiao, 2009) \\
\hline Cáscara de coco & $\mathrm{Cd}(\mathrm{II})$ & 37.78 & (Sousa et al., 2010) \\
\hline Hueso de olivo & $\mathrm{Cd}(\mathrm{II})$ & 7.73 & (Fiol et al., 2006) \\
\hline Paja de arroz & $\mathrm{Cd}(\mathrm{II})$ & 13.84 & (Ding, Jing, Gong, Zhou \& Yang, 2012) \\
\hline Cáscara de plátano & $\mathrm{Pb}(\mathrm{II})$ & 2.18 & (Anwar et al., 2010) \\
\hline Bagazo de agave & $\mathrm{Pb}(\mathrm{II})$ & 35.60 & (Velázquez et al., 2013) \\
\hline Césped de jardín & $\mathrm{Pb}(\mathrm{II})$ & 58.34 & (Hossain, Ngo, Guo \& Setiadi, 2012) \\
\hline Hueso de olivo & $\mathrm{Pb}(\mathrm{II})$ & 9.261 & (Fiol et al., 2006) \\
\hline Cáscara de mandarina & $\mathrm{Pb}(\mathrm{II})$ & 112.1 & (Pavan, Mazzocato, Jacques \& Dias, 2008) \\
\hline Mazorca de maíz & $\mathrm{Pb}(\mathrm{II})$ & 16.22 & (Tan et al., 2010) \\
\hline Aserrín & $\mathrm{Pb}(\mathrm{II})$ & 15.9 & (Bulut \& Tez, 2007) \\
\hline Cáscara de melón & $\mathrm{Pb}(\mathrm{II})$ & 167.8 & (Huang \& Zhu, 2013) \\
\hline Cáscara de ajo & $\mathrm{Pb}(\mathrm{II})$ & 51.73 & (Liu, Liu, Tao, Yu \& Jiang, 2014) \\
\hline Cáscara de coco & $\mathrm{Pb}(\mathrm{II})$ & 54.62 & (Sousa et al., 2010) \\
\hline Bagazo de agave & $\mathrm{Zn}(\mathrm{II})$ & 7.84 & (Velázquez et al., 2013) \\
\hline Cáscara de mango & $\mathrm{Zn}(\mathrm{II})$ & 28.21 & (Iqbal et al., 2009) \\
\hline Cáscara de kiwi & $\mathrm{Zn}(\mathrm{II})$ & 280 & (Al-Qahtani, 2016) \\
\hline Cáscara de plátano & $\mathrm{Zn}(\mathrm{II})$ & 125 & (Al-Qahtani, 2016) \\
\hline Cáscara de mandarina & $\mathrm{Zn}(\mathrm{II})$ & 260 & (Al-Qahtani, 2016) \\
\hline Aserrín & $\mathrm{Zn}(\mathrm{II})$ & 0.96 & (Šćiban, Radetic, Kevrešan \& Klašnja, 2007) \\
\hline Cáscara de coco & $\mathrm{Zn}(\mathrm{II})$ & 17.08 & (Sousa et al., 2010) \\
\hline Cáscara de lenteja & $\mathrm{Cu}(\mathrm{II})$ & 9.59 & (Aydin et al., 2008) \\
\hline Cáscara de mango & $\mathrm{Cu}(\mathrm{II})$ & 46.09 & (Iqbal et al., 2009) \\
\hline Paja de cebada & $\mathrm{Cu}(\mathrm{II})$ & 4.64 & (Pehlivan et al., 2012) \\
\hline Flores de sol & $\mathrm{Cu}(\mathrm{II})$ & 57.14 & (Witek-Krowiak, 2012) \\
\hline Paja de avena & $\mathrm{Cu}(\mathrm{II})$ & 17.42 & (Aydin et al., 2008) \\
\hline Cáscara de papa & $\mathrm{Cu}(\mathrm{II})$ & 0.3877 & (Aman, Kazi, Sabri \& Bano, 2008) \\
\hline Cáscara de arroz & $\mathrm{Cu}(\mathrm{II})$ & 2.95 & (Aydin et al., 2008) \\
\hline Hueso de olivo & $\mathrm{Cu}(\mathrm{II})$ & 2.027 & (Fiol et al., 2006) \\
\hline Cáscara de naranja & $\mathrm{Cu}(\mathrm{II})$ & 50.94 & (Sha \& Ningchuan, 2009) \\
\hline Cáscara de coco & $\mathrm{Cu}(\mathrm{II})$ & 41.36 & (Sousa et al., 2010) \\
\hline Aserrín & $\mathrm{Cu}(\mathrm{II})$ & 6.88 & (Šćiban et al., 2007) \\
\hline Cáscara de toronja & $\mathrm{Cu}(\mathrm{II})$ & 19.7 & (Tasaso, 2014) \\
\hline Cáscara de nuez de la india & $\mathrm{Ni}(\mathrm{II})$ & 18.86 & $\begin{array}{l}\text { (Kumar, Ramalingam, Kirupha, Murugesan, } \\
\text { Vidhyadevi \& Sivanesan, 2011) }\end{array}$ \\
\hline
\end{tabular}


Tabla IV. Capacidad máxima de bioadsorción para distintos subproductos agroindustriales (continuación).

\begin{tabular}{|c|c|c|c|}
\hline Biomasa & Adsorbato & $\begin{array}{l}\text { Capacidad máxima de } \\
\text { adsorción (mg/g) }\end{array}$ & Referencia \\
\hline Cáscara de mango & $\mathrm{Ni}(\mathrm{II})$ & 39.75 & (Iqbal et al., 2009) \\
\hline Cáscara de coco & $\mathrm{Ni}(\mathrm{II})$ & 16.34 & (Sousa et al., 2010) \\
\hline Hueso de olivo & $\mathrm{Ni}(\mathrm{II})$ & 2.13 & (Fiol et al., 2006) \\
\hline Aserrín & $\mathrm{Ni}(\mathrm{II})$ & 3.29 & (Bulut \& Tez, 2007) \\
\hline Cáscara de toronja & $\mathrm{Ni}(\mathrm{II})$ & 46.13 & $\begin{array}{l}\text { (Torab, Asadollahzadeh, Hemmati \& } \\
\text { Khosravi, 2013) }\end{array}$ \\
\hline Cáscara de mandarina & $\operatorname{Hg}(\mathrm{II})$ & 19.01 & (Husein, 2013) \\
\hline Bagazo de caña de azúcar & $\mathrm{Hg}(\mathrm{II})$ & 35.71 & (Khoramzadeh, Nasernejad \& Halladj, 2013) \\
\hline Cáscara de mandarina & $\mathrm{Cr}(\mathrm{VI})$ & 350 & (Al-Qahtani, 2016) \\
\hline Cáscara de lima & $\mathrm{Cr}(\mathrm{VI})$ & 250 & (Saha et al., 2013) \\
\hline Cáscara de plátano & $\mathrm{Cr}(\mathrm{VI})$ & 240 & (Al-Qahtani, 2016) \\
\hline Cáscara de arroz & $\mathrm{Cr}(\mathrm{VI})$ & 8.5 & (Bansal, Garg, Singh \& Garg, 2009) \\
\hline Orujo de oliva & $\mathrm{Cr}(\mathrm{VI})$ & 13.95 & $\begin{array}{l}\text { (Krishnani, Meng, Christodoulatos \& Boddu, } \\
\text { 2008) }\end{array}$ \\
\hline
\end{tabular}

Las exportaciones de la agroindustria de México han incrementado su participación en el mercado mundial, superando los 13 mil millones de dólares, actualmente es reconocido por su comercialización de productos como cerveza, jitomate, aguacate, tequila y mezcal a 150 países, la agroindustria mexicana está constituida por alrededor de 70 mil empresas que transforman aproximadamente la mitad de la producción del campo y procesan más de 100 productos agropecuarios (Agro Revista Industrial del Campo, 2016).

En 2006, la generación de residuos de cultivos primarios fue de 60.13 millones de toneladas de materia seca, y fueron: tallos de maíz, tallos de sorgo, bagazo de caña de azúcar, paja de trigo, paja de cebada y paja de algodón, mostrados en la Tabla V. Además de los residuos de cultivos primarios, existen los residuos de cultivos secundarios, subproductos de la agroindustria que los procesa. (Valdez V. et al., 2010).

Tabla V. Subproductos agrícolas y agroindustriales.

\begin{tabular}{|l|c|}
\hline \multicolumn{1}{|c|}{ Subproducto agrícola } & $\begin{array}{c}\text { Producción (Millones de } \\
\text { toneladas anuales) }\end{array}$ \\
\hline Paja de cebada & 0.87 \\
\hline Paja de frijol & 1.35 \\
\hline Paja de garbanzo & 0.16 \\
\hline
\end{tabular}

\begin{tabular}{|l|c|}
\hline \multicolumn{1}{|c|}{ Subproducto agrícola } & $\begin{array}{c}\text { Producción (Millones de } \\
\text { toneladas anuales) }\end{array}$ \\
\hline Paja de lenteja & 0.01 \\
\hline Paja de haba & 0.02 \\
\hline Paja de arroz & 0.34 \\
\hline Paja de trigo & 3.38 \\
\hline Paja de canola & 0.01 \\
\hline Paja de algodón & 0.45 \\
\hline Paja de maíz & 21.89 \\
\hline Paja de cártamo & 0.07 \\
\hline Paja de sorgo & 5.52 \\
\hline Paja de haba de soja & 0.08 \\
\hline Paja de tabaco & 0.02 \\
\hline Paja de caña de azúcar & 50.55 \\
\hline Paja de maní & 0.07 \\
\hline Subproducto agroindustrial & \\
\hline Mazorca de maíz & 21.89 \\
\hline Cáscara de arroz & 0.34 \\
\hline Cáscara de maní & 0.07 \\
\hline Bagazo de café & 1.49 \\
\hline Bagazo de agave & 1.22 \\
\hline Bagazo de maguey & 0.28 \\
\hline Bagazo de caña de azúcar & 50.55 \\
\hline & Valdez V. et al., 2010) \\
\hline
\end{tabular}




\section{Bioadsorbente:}

- Disponibilidad de materia prima

- Capacidad de adsorción

- Cinética de adsorción

- Costos de preparación

- Regeneración

- Disposición final
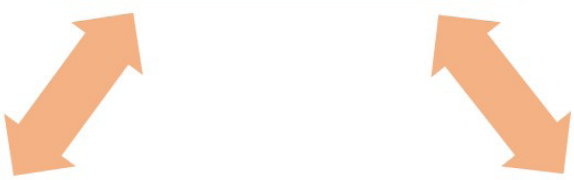

\section{Equipo de proceso:}

- Capacitación de operador

- Método batch/continuo

- Diseño del equipo

- Instrumentación y control

- Costos de operación y mantenimiento

- Espacio a ocupar.

Efluente:
- $\mathrm{pH}$
- Temperatura
- Sólidos suspendidos totales
- Especies químicas involucradas
- Otros contaminantes

Figura 3. Factibilidad de la bioadsorción utilizando subproductos agroindustriales. Elaboración personal.

En México, se generan anualmente cerca de 76 millones de toneladas de residuos orgánicos de frutas (limón, pera, manzana, papaya, piña, plátano y naranja) y vegetales (maíz, caña de azúcar, frijol, col, zanahoria, tomate, lechuga y papa) que generalmente no son aprovechados (González S. et al., 2015). En las agroindustrias dedicadas al procesamiento de cítricos, por lo general sólo se aprovecha una pequeña fracción comestible de la fruta, generando hasta un 50 por ciento de material residual, como cáscaras y semillas, dichos residuos solo son aprovechados para composteo (Narváez, 2018).

Una vez que se obtiene el bioadsorbente potencial, a partir de los resultados del laboratorio mediante pruebas de bioadsorción batch, que demuestra que el bioadsorbente tiene la capacidad para adsorber iones metálicos, se debe realizar un análisis tecnológico, para el escalamiento y/o utilización de efluentes reales, en la Figura 3, se muestran algunos puntos a considerar para su factibilidad tecnológica.

Es importante destacar, que es necesario seguir investigando métodos de desorción y disposición final de los bioadsorbentes saturados, ya que es fundamental para su aplicación a escala industrial y su cumplimiento con leyes y normas ambientales, además, se puede combinar con procesos convencionales de tratamiento de aguas residuales, para un mejor tratamiento de efluentes contaminados.

\section{Conclusiones}

El proceso de bioadsorción ofrece un método eficaz de remoción de metales pesados presentes en efluentes contaminados, en especial utilizando subproductos agroindustriales que proporcionan la ventaja de utilizarlos como un bioadsorbente de bajo costo con aplicación para aguas contaminadas $e . g$. aguas residuales industriales. México tiene potencial en la producción de bioadsorbentes, ya que genera una gran cantidad de residuos agroindustriales que no son aprovechados para la generación de nuevos productos. Es necesario realizar estudios de bioadsorción con diversos subproductos agroindustriales de gran generación que presentan un problema medioambiental, así como llevar a cabo pruebas a escala piloto industrial, para una aplicación real, esto involucra investigaciones como: preparación del bioadsorbente mediante granulación o aglomeración por fluidización, bioadsorción empleando reactores continuos (e. g. lecho empacado, lecho fluidizado, Air-lift, y sus distintas configuraciones de alimentación), utilización de efluentes industriales, estudios de regeneración y disposición final del bioadsorbente.

\section{Agradecimientos}

Jonathan M. Sanchez Silva agradece al Tecnológico Nacional de México/Instituto Tecnológico de Tepic por las facilidades para la realización de la Tesis profesional y al Consejo Estatal de Ciencia y Tecnología (COCYTEN) por la beca otorgada para la titulación. 


\section{REFERENCIAS}

Abbas, S., Ismail, I., Mostafa, T. \& Sulaymon, A. (2014). Biosorption of Heavy Metals: a Review. Journal of Chemical Science and Technology, 3, 74-102.

Abdolali, A., Guo, W. S., Ngo, H. H., Chen, S. S., Nguyen, N. C. \& Tung, K. L. (2014). Typical lignocellulosic wastes and by-products for biosorption process in water and wastewater treatment: A critical review. Bioresource Technology, 160, 57-66. DOI:10.1016/j. biortech.2013.12.037

Abdullah, N., Yusof, N., Lau, W., Jaafar, J. \& Ismail, A. (2019). Recent trends of heavy metal removal from water/wastewater by membrane technologies. Journal of Industrial and Engineering Chemistry, 76, 17-38. DOI:10.1016/j.jiec.2019.03.029

Acosta, I., López, V., Coronado, E., Cárdenas, J. F. \& Mártinez , V. M. (2010). Remoción de cromo (VI) en solución acuosa por la biomasa de cáscara de tamarindo (Tamarindus indica). BioTecnologia, 14(3), 11-17.

Aderhold, D., Williams, C. J. \& Edyvean, R. G. (1996). The removal of heavy-metal ions by seaweeds and their derivatives. Bioresource Technology, 58(1), 1-6. DOI:10.1016/S0960-8524(96)00072-7

Agro Revista Industrial del Campo. (2016, Octubre 6). 2000 AGRO Revista Industrial del Campo. Retrieved from http://www.2000agro.com.mx/agroindustria/ agroindustria-mexicana/

Ahalya, N., Kanamadi, R. \& Ramachandra, T. (2006). Biosorption of iron (III) from aqueous solutions using the husk of Cicer arietinum. Indian Journal of Chemical Technology, 13, 122-127.

Ahmet, S., Özgur, D. U. \& Tüzen, M. (2011). Equilibrium, thermodynamic and kinetic investigations on biosorption of arsenic from aqueous solution by algae (Mangeotia genuflexa) biomass. Chemical Engineering Journal, 167, 155-161. DOI:10.1016/j.cej.2010.12.014

Akpor, O., Ohiobor, G. \& Olaolu, T. (2014). Heavy metal pollutants in wastewater effluents: Sources effects and remediation. Advances in Bioscience and Bioengineering, 2(4), 37-43. DOI:10.11648/j.abb.20140204.11

Aksu, Z. (2005). Application of biosorption for the removal of organic pollutants: a review. Process Biochemistry, 40, 997-1026. DOI:10.1016/j.procbio.2004.04.008

Alfarra, A., Frackowiak, E. \& Béguin, F. (2004). The HSAB concept as a means to interpret the adsorption of metal ions onto activated carbons. Applied Surface Science, 228, 84-92. DOI:10.1016/j.apsusc.2003.12.033

Allard, B., Hakansson, K. \& Karlsson, S. (2005). The importance of sorption phenomena in relation to trace element speciation and mobility. Lecture Notes in Earth Sciences, 99-112. DOI:10.1007/BFb0019696

Alomá, I., Martín-Lara, M., Rodríguez, I., Blázquez, G. \& Calero, M. (2012). Removal of nickel (II) ions from aqueous solutions by biosorption on sugarcane bagasse.
Journal of the Taiwan Institute of Chemical Engineers, 43(2), 275-281. DOI:10.1016/j.jtice.2011.10.011

Al-Qahtani, K. M. (2016). Water purification using different waste fruit cortexes for the removal of heavy metals. Journal of Taibah University for Science, 10, 700-708. DOI:10.1016/j.jtusci.2015.09.001

Aman, T., Kazi, A. A., Sabri, M. U. \& Bano, Q. (2008). Potato peels as solid waste for the removal of heavy metal copper(II) from waste water/industrial effluent. Colloids and Surfaces B: Biointerfaces, 63(1), 116-121. DOI:10.1016/j.colsurfb.2007.11.013

Amaral, A., Cruz, J., Cunha, R. \& Rodrigues, A. (2006). Baseline Levels of Metals in Volcanic Soils of the Azores (Portugal). Soil \& Sediment Contamination, 15, 123-130. DOI:10.1080/15320380500506255

Ansari, M. I. \& Malik, A. (2007). Biosorption of nickel and cadmium by metal resistant bacterial isolates from agricultural soil irrigated with industrial wastewater. Bioresource Technology, 98(16), 3149-3153. DOI:10.1016/j.biortech.2006.10.008

Anwar, J., Shafique, U., Zaman, W.-u., Salman, M., Dar, A. \& Shafique, A. (2010). Removal of $\mathrm{Pb}(\mathrm{II})$ and $\mathrm{Cd}$ (II) from water by adsorption on peels of banana. Bioresource Technology, 101, 1752-1755. DOI:10.1016/j. biortech.2009.10.021

Asadi, F., Shariatmadari, H. \& Mirghaffari, N. (2008). Modification of rice hull and sawdust sorptive characteristics for remove heavy metals from synthetic solutions and wastewater. Journal of Hazardous Materials, 154(1-3), 451-458. DOI:10.1016/j.jhazmat.2007.10.046

Aydin, H., Bulut, Y. \& Yerlikaya, Ç. (2008). Removal of copper (II) from aqueous solution by adsorption onto lowcost adsorbents. Journal of Environmental Management, 87, 37-45. DOI:10.1016/j.jenvman.2007.01.005

Bankar , A. \& Nagaraja, G. (2018). Recent Trends in Biosorption of Heavy Metals by Actinobacteria. In B. Pratap Singh, A. Kumar Passari \& V. Gupta Kumar, New and Future Developments in Microbial Biotechnology and Bioengineering; Actinobacteria: Diversity and Biotechnological Applications (pp. 257-275). Elsevier. DOI:10.1016/B978-0-444-63994-3.00018-7

Bansal, M., Garg, U., Singh, D. \& Garg, V. (2009). Removal of $\mathrm{Cr}(\mathrm{VI})$ from aqueous solutions using pre-consumer processing agricultural waste: A case study of rice husk. Journal of Hazardous Materials, 162(1), 312-320. DOI:10.1016/j.jhazmat.2008.05.037

Baysal, Z., Cinar, E., Bulut, Y., Alkan, H. \& Dogru, M. (2009). Equilibrium and thermodynamic studies on biosorption of $\mathrm{Pb}(\mathrm{II})$ onto Candida albicans biomass. Journal of Hazardous Materials, 161(1), 62-67. DOI:10.1016/j. jhazmat.2008.02.122

Bhan, A. \& Sarkar, N. (2004). Mercury in the Environment: Effect on Health and Reproduction. Reviews on environmental health, 20(1), 39-56. DOI:10.1515/ 
REVEH.2005.20.1.39

Bhatnagar, A. \& Minocha, A. (2010). Biosorption optimization of nickel removal from water using Punica granatum peel waste. Colloids and Surfaces B: Biointerfaces, 76(2), 544-548. DOI:10.1016/j.colsurfb.2009.12.016

Bhatnagar, A. \& Sillanpaa, M. (2010). Utilization of agroindustrial and municipal waste materials as potential adsorbents for water treatment-A review. Chemical Engineering Journal, 157(2-3), 288-296. DOI:10.1016/j. cej.2010.01.007

Bhatnagar, A., Sillanpaa, M. \& Witek-Krowiak, A. (2015). Agricultural waste peels as versatile biomass for water purification - A review. Chemical Engineering Journal, 270, 244-271. DOI:10.1016/j.cej.2015.01.135

Bonilla-Petriciolet, A., Mendoza-Castillo, D. I. \& Reynel, H. E. (2017). Adsorption Processes for Water Treatment and Purification. Chemistry Molecular Sciences and Chemical Engineering, 1-21. DOI:10.1016/B978-0-12409547-2.14390-2

Bulut, Y. \& Tez, Z. (2007). Removal of heavy metals from aqueous solution by sawdust adsorption. Journal of Environmental Sciences, 19(2), 160-166. DOI:10.1016/ S1001-0742(07)60026-6

Burakov, A., Galumin, E., Bukarova, I., Kucherova, A., Agarwal, S., Tkachev, A. \& Gupta, V. (2018). Adsorption of heavy metals on conventional and nanostructured materials for wastewater treatment purposes: A review. Exotoxicology and Environmental Safety, 148, 702-712. DOI:10.1016/j.ecoenv.2017.11.034

Casas G, L. \& Sandoval F, G. (2014). Enzimas en la valorización de residuos agroindustriales. Revista digital universitaria, 15(12), 1-15.

Covarrubias, S. A. \& Peña Cabriales, J. J. (2017). Contaminación ambiental por metales pesados en méxico: Problematica y estrategias de fitoremediación. Revista Internacional de Contaminación Ambiental, 33, 7-21. DOI:10.20937/RICA.2017.33.esp01.01

Crini, G. (2005). Recent developments in polysaccharidebased materials used as adsorbents in wastewater treatment. Progress in Polymer Science, 30(1), 38-70. DOI:10.1016/j.progpolymsci.2004.11.002

Cury, R., K., Aguas, M., Y., Martínez M., A., Olivero R., R. \& Chams Ch., L. (2017). Residuos agroindustriales su impacto, manejo y aprovechamiento. Revista Colombiana de Ciencia Animal, 9, 122-132. DOI:10.24188/recia. v9.nS.2017.530

Davis, T., Volesky, B. \& Mucci, A. (2003). A review of the biochemistry of heavy metal biosorption by brown algae. Water Research, 37(18), 4311-4330. DOI:10.1016/ S0043-1354(03)00293-8

Davis, T. A., Volesky, B. \& Vieira, R. (2000). Sargassum seawed as biosorbent for heavy metals. Water Research, 34, 4270-4278. DOI:10.1016/S0043-1354(00)00177-9

Dhankhar, R. \& Hooda, A. (2011). Fungal biosorption - an alternative to meet the challenges of heavy metal pollution in aqueous solutions. Environmental Technology, 32(5), 467-491. DOI:10.1080/09593330.2011.572922

Ding, Y., Jing, D., Gong, H., Zhou, L. \& Yang, X. (2012). Biosorption of aquatic cadmium(II) by unmodified rice straw. Bioresource Technology, 114, 20-25. DOI:10.1016/j.biortech.2012.01.110

Diniz , V., Weber, M., Volesky, B. \& Naja, G. (2008). Column biosorption of lanthanum and europium by sargassum. Water Research, 42, 363-371. DOI:10.1016/j. watres.2007.07.027

Dursun, A. Y. (2006). A comparative study on determination of the equilibrium, kinetic and thermodynamic parameters of biosorption of copper(II) and lead(II) ions onto pretreated Aspergillus niger. Biochemical Engineering Journal, 28(2), 187-195. DOI:10.1016/j.bej.2005.11.003

Eccles, H. (1999). Treatment of metal-contaminated wastes: Why select a biological process? Trends in Biotechnology, 17(12), 462-465. DOI: 10.1016/S0167-7799(99)01381-5

Enniya, I., Rghioui, L. \& Jourani, A. (2018). Adsorption of hexavalent chromium in aqueous solution on activated carbon prepared from apple peels. Sustaintable Chemistry and Pharmacy, 7, 9-16. DOI:10.1016/j.scp.2017.11.003

Farooq, U., Kozinski, J. A., Khan, M. A. \& Athar, M. (2010). Biosorption of heavy metal ions using wheat based biosorbents - A review of the recent literature. Bioresource Technology, 101(14), 5043-5053. DOI:10.1016/j. biortech.2010.02.030

Fiol, N., Villaescusa, I., Martínez, M., Miralles, N., Poch, J. \& Serarols, J. (2006). Sorption of $\mathrm{Pb}(\mathrm{II}), \mathrm{Ni}(\mathrm{II}), \mathrm{Cu}(\mathrm{II})$ and $\mathrm{Cd}(\mathrm{II})$ from aqueous solution by olive stone waste. Separation and Purification Technology, 50(1), 132-140. DOI:10.1016/j.seppur.2005.11.016

Gadd, G. (2008). Biosorption: critical review of scientific rationale,environmental importance and significance for pollution treatment. Journal of Chemical Technology and Biotechnology, 84, 13-28. DOI:10.1002/jctb.1999

Gautam, R. K., Mudhoo, A., Lofrano, G. \& Chattopadhyaya, M. C. (2014). Biomass-derived biosorbents for metal ions sequestration: Adsorbent modification and activation methods and adsorbent regeneration. Journal of Environmental Chemical Engineering, 2(1), 239-259. DOI:10.1016/j.jece.2013.12.019

Gisi, S. D., Lofrano, G., Grassi, M. \& Notarnicola, M. (2016). Characteristics and adsorption capacities of low-cost sorbents for wastewater treatment: A review. Sustainable Materials and Technologies, 9, 10-40. DOI:10.1016/j. susmat.2016.06.002

González S., M. E., Pérez F., S., Wong W., A., Bello M., R. \& Yañez O., G. (2015). Residuos agroindustriales con potencial para la producción de metano mediante la digestión anaerobia. Revista Argentina de Microbiología, 47(3), 229-235. DOI:10.1016/j.ram.2015.05.003

Grassi, M., Kaykioglu, G., Belgiorno, V. \& Lofrano, G. 
(2012). Removal of Emerging Contaminants from Water and Wastewate by Adsorption Process. In G. Lofrano, Emerging Compounds Removal from Wastewater: Natural and Solar Based Treatments (pp. 29-51). Italia: Springer. DOI:10.1007/978-94-007-3916-1

Greene, B., Henzl, M., Hosea, J. \& Darnall, D. (1986). Elimination of Bicarbonate Intederence in the Binding of $\mathrm{U}(\mathrm{Vl})$ in Mill-Waters to Freeze-dried Chlorella vulgaris. Biotechnology and Bioengineering, 18, 764-767. DOI:10.1002/bit.260280519

Gu, J., Liang, J., Chen, C., Li, K., Zhou, W., Jia, J. \& Sun, T. (2020). Treatment of real deplating wastewater through an environmental friendly precipitation-electrodepositionoxidation process: Recovery of silver and copper and reuse of wastewater. Separation and Purification Technology, 248, 117082. DOI: 10.1016/j.seppur.2020.117082

Gupta, V. K., Rastogi, A. \& Nayak, A. (2010). Biosorption of nickel onto treated algae (Oedogonium hatei): Application of isotherm and kinetic models. Journal of Colloid and Interface Science, 342(2), 533-539. DOI:10.1016/j. jcis.2009.10.074

Hlihor, R. M., Bulgariu, L., Sobariu, D. L., Diaconu, M., Tavares, T. \& Gavrilescu, M. (2014). Recent advances in biosorption of heavy metals: support tools for biosorption equilibrium, kinetics and mechanism. Revue Roumaine de Chimie, 59, 527-238.

Hossain, M., Ngo, H., Guo, W. \& Setiadi, T. (2012). Adsorption and desorption of copper(II) ions onto garden grass. Bioresource Technology, 121, 386-395. DOI:10.1016/j. biortech.2012.06.119

Huang, K. \& Zhu, H. (2013). Removal of Pb2+ from aqueous solution by adsorption on chemically modified muskmelon peel. Environmental Science and Pollution Research, 20(7), 4424-4434. DOI:10.1007/s11356-0121361-7

Husein, D. Z. (2013). Adsorption and removal of mercury ions from aqueous solution using raw and chemically modified Egyptian mandarin peel. Desalination and Water Treatment, 51(34-36), 6761-6769. DOI:10.1080/1 9443994.2013.801793

Iqbal, M., Saeed, A. \& Kalim, I. (2009). Characterization of Adsorptive Capacity and Investigation of Mechanism of $\mathrm{Cu} 2^{+}, \mathrm{Ni}_{2}{ }^{+}$and $\mathrm{Zn} 2^{+}$Adsorption on Mango Peel Waste from Constituted Metal Solution and Genuine Electroplating Effluent. Separation Science and Technology, 44(15), 3770-3791. DOI:10.1080/01496390903182305

Javanbakht, V., Alavi Amir, S. \& Zilouei, H. (2014). Mechanisms of heavy metal removal using microorganisms as biosorbent. Water Science \& Technology, 69(9), 17751787. DOI:10.2166/wst.2013.718

Jobby, R., Jha, P., Yadav Kumar, A. \& Desai, N. (2018). Biosorption and biotransformation of hexavalent Chromium [Cr (VI)]: A comprehensive review. Chemosphere, 207, 255-266. DOI:10.1016/j. chemosphere.2018.05.050

Kaizer, A. \& Osakwe, S. (2010). Physicochemical characteristics and heavy metal levels in water samples from five river systems in Delta State, Nigeria. J. Applicat. Sci. Environ. Manage, 14(1), 83-87. DOI:10.4314/jasem. v14i1.56501

Khoramzadeh, E., Nasernejad, B. \& Halladj, R. (2013). Mercury biosorption from aqueous solutions by Sugarcane Bagasse. Journal of the Taiwan Institute of Chemical Engineers, 44(2), 266-269. DOI:10.1016/j. jtice.2012.09.004

Khosa, M. \& Ullah, A. (2018). Mechanistic insight into protein supported biosorption complemented by kinetic and thermodynamics perspectives. Advances in Colloid and Interfance Science, 261, 28-40. DOI:10.1016/j. cis.2018.09.004

Krishnani, K. K., Meng, X., Christodoulatos, C. \& Boddu, V. M. (2008). Biosorption mechanism of nine different heavy metals onto biomatrix from rice husk. Journal of Hazardous Materials, 153(3), 1222-1234. doi:10.1016/j. jhazmat.2007.09.113

Kumar, P. S., Ramalingam, S., Kirupha, S. D., Murugesan, A., Vidhyadevi, T. \& Sivanesan, S. (2011). Adsorption behavior of nickel(II) onto cashew nut shell: Equilibrium, thermodynamics, kinetics, mechanism and process design. Chemical Engineering Journal, 167(1), 122-131. DOI:10.1016/j.cej.2010.12.010

Kurniawan, T. A., Chan, G., Lo, W.-H. \& Babel, S. (2006). Physico-chemical treatment techniques for wastewater laden with heavy metals. Chemical Engineering Journal, 118, 83-98. DOI:10.1016/j.cej.2006.01.015

Kuyucak, N. \& Volesky, B. (1989). The mechanism of cobalt biosorption. Biotechnology and Bioengineering, 33, 823831. DOI:10.1002/bit.260330705

Liu, W., Liu, Y., Tao, Y., Yu, Y. \& Jiang, H. (2014). Comparative study of adsorption of $\mathrm{Pb}$ (II) on native garlic peel and mercerized garlic peel. Environmental Science and Pollution Research, 21(3), 2054-2063. DOI:10.1007/ s11356-013-2112-0

Liu, Z., Wang, L.-a., Xiao, H., Guo, X., Urbanovich, O., Nagorskaya, L. \& Li, X. (2020). A review on control factors of pyrolysis technology for plants containing heavy metals. Ecotoxicology and Environmental Safety, 191, 110-181. doi:10.1016/j.ecoenv.2020.110181

Malik, A. (2004). Metal bioremediation through growing cells. Environment International, 30(2), 261-278. DOI:10.1016/j.envint.2003.08.001

Mclean, R., Fortin, D. \& Brown, D. (2011). Microbial metalbinding mechanisms and their relation to nuclear waste disposal. Canadian Journal of Microbiology, 42, 392400. DOI:10.1139/m96-055

Mendoza Castillo, D. (2012). Sorción multicomponente de metales pesados en agua empleando carbón de hueso. Tesis para obtener el grado de Doctora en Ciencias 
Biológicas, 100-101.

Michalak , I., Chojnacka, K. \& Witek Krowiak, A. (2013). State of the Art for the Biosorption Process - a Review. Applied Biochemistry and Biotechnology, 170, 13891416. DOI:10.1007/s12010-013-0269-0

Miretzky, P. \& Fernandez Cirelli, A. (2010). Cr(VI) and $\mathrm{Cr}$ (III) removal from aqueous solution by raw and modified lignocellulosic materials: A review. Journal of Hazardous Materials, 180, 1-19. DOI:10.1016/j. jhazmat.2010.04.060

Montazer-Rahmati, M., Rabbani, P., Abdolali, A. \& Keshtkar, A. R. (2011). Kinetics and equilibrium studies on biosorption of cadmium, lead, and nickel ions from aqueous solutions by intact and chemically modified brown algae. Journal of Hazardous Materials, (185), 401-407. DOI:10.1016/j.jhazmat.2010.09.047

Naja, G. \& Volesky, B. (2011). Chapter 3: The Mechanism of Metal Cation and Anion Biosorption. In P. kotrba, M. Mackova \& T. Macek, Microbial Biosorption of Metals (pp. 19-58). Springer. DOI:10.1007/978-94-007-0443-5

Naja, G., Murphy, V. \& Volesky, B. (2010). Biosorption, Metals. Encyclopedia of Industrial Biotechnology: Bioprocess, Bioseparation, and Cell Technology, 29. DOI:10.1002/9780470054581.eib166.

Narváez, M. (2018, Diciembre 5). Cienciamx. Retrieved from www.cienciamx.com/index.php/ciencia/quimica/23762aprovechamiento-residuos-agroindustriales-citricos

Naseem, R. \& Tahir, S. S. (2001). Removal of Pb(II) from aqueous/acidic solutions by using bentonite as an adsorbent. Water Research, 35(16), 3982-3986. DOI:10.1016/S0043-1354(01)00130-0

Nijkam, E. \& Schiewer, S. (2012). Optimization and kinetic modeling of cadmium desorption from citrus peels: A process for biosorbent regeneration. Journal of Hazardous Materials, 213-214, 242-248. DOI:10.1016/j. jhazmat.2012.01.084

Nordberg, G., Herber, R. \& Alessio, L. (1992). Cadmium in the Human Environment: Toxicity and Carcinogenicity. IARC Scientific Publications, 469.

O'Connell, D. W., Birkinshaw, C. \& O'Dwyer, T. (2008). Heavy metal adsorbents prepared from the modification of cellulose: A review. Bioresource Technology, 99(15), 6709-6724. DOI:10.1016/j.biortech.2008.01.036

Ogura, H., Takeuchi, T. \& Morimoto, K. (1996). A comparison of the 8-hydroxydeoxyguanosine, chromosome aberrations and micronucleus techniques for the assessment of the genotoxicity of mercury compounds in human blood lymphocytes. Mutation Research/Reviews in Genetic Toxicology, 340, 175-182. DOI:10.1016/ S0165-1110(96)90047-0

Panigatti, M., Torres, J., Griffa, C., Boglione, R. \& Gentinetta, F. (2007). Biorremediación de efluentes con cromo (VI) proveniente de plantas metalmecanicas. Revista de Ingeniería y Ciencias Ambientales: Investigación, desarrollo y práctica. (AIDIS), 1(2)

Park, D., Yun, Y.-S. \& Park, J. M. (2010). The past, present, and future trends of biosorption. Biotechnology and Bioprocess Engineering, 15(1), 86-102. DOI:10.1007/ s12257-009-0199-4

Pavan, F. A., Mazzocato, A. C., Jacques, R. A. \& Dias, S. L. (2008). Ponkan peel: A potential biosorbent for removal of $\mathrm{Pb}$ (II) ions from aqueous solution. Biochemical Engineering Journal, 40(2), 357-362. DOI:10.1016/j. bej.2008.01.004

Pehlivan, E., Altun, T. \& Parlayici, S. (2012). Modified barley straw as a potential biosorbent for removal of copper ions from aqueous solution. Food Chemistry, 135(4), 22292234. DOI:10.1016/j.foodchem.2012.07.017

Pellera, F., Giannis, A., Kalderis, D., Anastasiadou, K., Stegmann, R., Wang, J.-Y. \& Gidarakos, E. (2012). Adsorption of $\mathrm{Cu}(\mathrm{II})$ ions from aqueous solutions on biochars prepared from agricultural by-products. Journal of Environmental Management, 96(1), 35-42. DOI:10.1016/j.jenvman.2011.10.010

Poots , V., McKay, G. \& Healy, J. (1976). The removal of acid dye from effluent using natural adsorbents - I Peat. Water Research, 10, 1061-1066. DOI:10.1016/00431354(76)90036-1

Rangabhashiyam, S. \& Balasubramanian, P. (2019). Characteristics, perfomances, equilibrium and kinetic modeling aspects of heavy metal removal using algae. Bioresource Technology Reports, 5, 261-279. DOI:10.1016/j.biteb.2018.07.009

Rangabhashiyam, S., Lata, S. \& Balasubramanian, P. (2018). Biosorption characteristics of methylene blue and malachite green from simulated wastewater onto Carica papaya wood biosorbent. Surfaces and Interfaces, 10, 197-215. doi:10.1016/j.surfin.2017.09.011

Rangabhashiyam, S., Selvaraju, S., Selvaraju, N. \& Varguese, L. A. (2014). Significance of exploiting non-living biomaterials for the biosorption of wastewater pollutants. World Journal of Microbiology and Biotechnology, 30(6), 1-21. DOI:10.1007/s11274-014-1599-y

Rao, L. N. \& Prabhakar, G. (2011). Removal of heavy metals by biosorption - an overall review. Journal of Engineering Research and Studies, 2(4), 7-22.

Rao, K., Mohapatra, M., Anand, S. \& Venkateswarlu, P. (2010). Review on cadmium removal from aqueous solutions. International Journal of Engineering, Science and Technology, 2(7), 81-103. DOI:10.4314/ijest. v2i7.63747

Rocha, C. G., Morozin Z, D. A., Ventura da Silva A., R. \& Alberto da Silva A., A. A. (2009). Use of rice straw as biosorbent for removal of $\mathrm{Cu}(\mathrm{II}), \mathrm{Zn}$ (II), $\mathrm{Cd}(\mathrm{II})$ and $\mathrm{Hg}(\mathrm{II})$ ions in industrial effluents. Journal of Hazardous Materials, 166(1), 383-388. DOI:10.1016/j. jhazmat.2008.11.074

Rubio, J., Souza, M. L. \& Smith, R. W. (2002). Overview of 
flotation as a wastewater treatment technique. Minerals Enginerring, 15(3), 139-155. DOI: 10.1016/s08926875(01)00216-3

Sag, Y. \& Kursal, T. (2001). Recent Trends in the Biosorption of Heavy Metals: A Review. Biotechnology and Bioprocess Enginnering, 6(6), 376-385. DOI:10.1007/BF02932318

Saha, R., Mukherjee, K. \& Saha, I. (2013). Removal of hexavalent chromium from water by adsorption on mosambi (Citrus limetta) peel. Research on Chemical Intermediates, 39(5), 2245-2257. DOI:10.1007/s11164012-0754-z

Salvador, F., Martín-Sánchez, N., Sánchez-Hernández, R., Sánchez-Montero, M. J. \& Izquierdo, C. (2015a). Regeneration of carbonaceous adsorbents. Part I: Thermal Regeneration. Microporous and Mesoporous Materials, 202, 259-276. DOI:10.1016/j.micromeso.2014.02.045

Salvador, F., Martín S. N., Sánchez, H. R., Sánchez, M. J. \& Izquierdo, C. (2015b). Regeneration of carbonaceous adsorbents. PartII: Chemical, Microbiological and Vacuum Regeneration. Microporous and Mesoporous Materials, 202, 277-296. DOI:10.1016/j.micromeso.2014.08.019

Sanfeliu, C., Sebastia, J., Cristófol, R. \& Rodriguez-Farre, E. (2003). Neurotoxicity of organomercurial compounds. Neurotoxicity Research, 5(4), 283-305. DOI:10.1007/ BF03033386

Santos, S., Ungureanu, G., Volf, I., Boaventura, R. \& Botelho, C. (2018). Macroalgae Biomass as Sorbent for Metal Ions. In V. Popa, I. Volf \& Elsevier (Ed.), Biomass as Renewable Raw Material to Obtain Bioproducts of HighTech Value (pp. 69-112). Elsevier. DOI:10.1016/B978-0444-63774-1.00003-X

Sardar, K., Ali, S., Hammed, S., Afzal, S., Fatima, S., Bilal, S. M., Aslam, B. S. \& Tauqeer, H. M. (2013). Heavy Metals Contamination and what are the Impacts on Living Organisms. Greener Journal of Environment Management and Public Safety, 2(4), 172-179. DOI:10.15580/ GJEMPS.2013.4.060413652

Schoeters, G., Hond, E., Zuurbier, M., Naginiene, R., Van Den Hazel, P., Stilianakis, N., Ronchetti, R. \& Koppe, J. G. (2008). Cadmium and children: Exposure and health effects. Acta Paediatrica, 95, 50-54. DOI:10.1080/08035320600886232

Šćiban, M., Radetic, B., Kevrešan, Ž. \& Klašnja, M. (2007). Adsorption of heavy metals from electroplating wastewater by wood sawdust. Bioresource Technology, 98(2), 402-409. DOI:10.1016/j.biortech.2005.12.014

Sha, L. X. \& Ningchuan, F. Q. (2009). Adsorption of $\mathrm{Cu}^{+}$ and $\mathrm{Cd}^{+}$from aqueous solution by mercapto-acetic acid modified orange peel. Colloids and Surfaces B: Biointerfaces, 73(1), 10-14. DOI:10.1016/j. colsurfb.2009.04.021

Sousa, F. W., Oliveira, A. G., Ribeiro, J. P., Rosa, M. F., Keukeleire, D. \& Nascimento, R. F. (2010). Green coconut shells applied as adsorbent for removal of toxic metal ions using fixed-bed column technology. Journal of Environmental Management, 91(8), 1634-1640. DOI:10.1016/j.jenvman.2010.02.011

Sutherland, C. \& Venkobachar, C. (2010). A diffusionchemisorption kinetic model for simulating biosorption using forest macro-fungus, fomes fasciatus. International Research Journal of Plant Science, 1(4), 107-117.

Svecova, L., Spanelova, M., Kubal, M. \& Guibal, E. (2006). Cadmium, lead and mercury biosorption on waste fungal biomass issued from fermentation industry. I. Equilibrium studies. Separation and Purification Technology, 52, $142-$ 153. DOI:10.1016/j.seppur.2006.03.024

Tan, G. \& Xiao, D. (2009). Adsorption of cadmium ion from aqueous solution by ground wheat stems. Journal of Hazardous Materials, 164(2-3), 1359-1363. DOI:10.1016/j.jhazmat.2008.09.082

Tan, G., Yuan, H., Liu, Y. \& Xiao, D. (2010). Removal of lead from aqueous solution with native and chemically modified corncobs. Journal of Hazardous Materials, 174(1-3), 740-745. DOI:10.1016/j.jhazmat.2009.09.114

Tasaso, P. (2014). Adsorption of Copper Using Pomelo Peel and Depectinated Pomelo Peel. Clean Energy Technology, 2, 154-157. DOI:10.7763/JOCET.2014.V2.112

Tejada Tovar, C., Villabona Ortiz, Á. \& Garcés Jaraba, L. (2015). Adsorción de metales pesados en aguas residuales usando materiales de origen biológico. TecnoLógicas, 18(34), 109-123. DOI:10.22430/22565337.209

Torab, M. M., Asadollahzadeh, M., Hemmati, A. \& Khosravi, A. (2013). Equilibrium, kinetic, and thermodynamic studies for biosorption of cadmium and nickel on grapefruit peel. Journal of the Taiwan Institute of Chemical Engineers, 44(2), 295-302. DOI:10.1016/j. jtice.2012.11.001

Uluozlu, O., Sari, A., Tuzen, M. \& Soylak, M. (2008). Biosorption of $\mathrm{Pb}(\mathrm{II})$ and $\mathrm{Cr}$ (III) from aqueous solution by lichen (Parmelina tiliaceae) biomass. Bioresource Techonology, 99, 2972-2980. DOI:10.1016/j. biortech.2007.06.052

USEPA, U. S. (2007). Framework for Metals Risk Assessment. Washington.

Valdez, V. I., Acevedo B., J. A. \& Hernández S., C. (2010). Distribution and potential of bioenergy resources from agricultural activities in Mexico. Renewable and Sustainable Energy Reviews, 14, 2147-2153. DOI:10.1016/j.rser.2010.03.034

Valdman, E., Erijman, L., Pessoa, F. \& Leite, S. (2001). Continuous biosorption of $\mathrm{Cu}$ and $\mathrm{Zn}$ by immobilized waste. Process Biochemistry, 36, 869-873. DOI:10.1016/ S0032-9592(00)00288-0

Valko, M., Rhodes, C., Moncol, J., Izakovic, M. \& Mazur, M. (2006). Free radicals, metals and antioxidants in oxidative stress-induced cancer. Chemico-Biological Interactions, 160(1), 1-40. DOI:10.1016/j.cbi.2005.12.009

Vardhan, K., Kumar, P. \& Panda, R. (2019). A review on heavy 
metal pollution, toxicity and remedial measures: Current trends and future perspectives. Journal of Molecular Liquids, 290, 111197 DOI:10.1016/j.molliq.2019.111197

Velázquez Jiménez, L., Pavlick, A. \& Rangel Méndez, J. (2013). Chemical characterization of raw and treated agave bagasse and its potential as adsorbent of metal cations from water. Industrial Crops and Products, 43, 200-206. DOI:10.1016/j.indcrop.2012.06.049

Vijayaraghavan, K. \& Yun, Y.-S. (2008). Bacterial biosorbents and biosorption. Biotechnology Advances, 26(3), 266291. DOI:10.1016/j.biotechadv.2008.02.002
Volesky, B. (2007). Biosorption and me. Water Research, 41, 4017-4029. DOI:10.1016/j.watres.2007.05.062

Witek-Krowiak, A. (2012). Analysis of temperature-dependent biosorption of $\mathrm{Cu} 2^{+}$ions on sunflower hulls: Kinetics, equilibrium and mechanism of the process. Chemical Engineering Journal, 192(1), 13-20. DOI:10.1016/j. cej.2012.03.075

Won, S., Han, M. \& Yun, Y. (2008). Different blinding mechanisms in biosorption of reactive dyes according to their reactivity. Water Research, 42, 4847-4855. DOI:10.1016/j.watres.2008.09.003 